\title{
On a Subclass of Analytic Functions That Are Starlike with Respect to a Boundary Point Involving Exponential Function
}

\author{
Adam Lecko $\mathbb{D}^{1},{ }^{1}$ Gangadharan Murugusundaramoorthy $\mathbb{D}^{2}{ }^{2}$ \\ and Srikandan Sivasubramanian (D) $^{3}$ \\ ${ }^{1}$ Department of Complex Analysis, Faculty of Mathematics and Computer Science, University of Warmia and Mazury in Olsztyn, \\ ul. SÅ,onecz na 54, 10-710 Olsztyn, Poland \\ ${ }^{2}$ Department of Mathematics, School of Advanced Sciences, Vellore Institute of Technology (Deemed to be University), \\ Vellore 632014, India \\ ${ }^{3}$ Department of Mathematics, University College of Engineering Tindivanam, Anna University, Tindivanam 604001, India
}

Correspondence should be addressed to Srikandan Sivasubramanian; sivasaisastha@rediffmail.com

Received 17 September 2021; Accepted 10 December 2021; Published 11 January 2022

Academic Editor: Mohsan Raza

Copyright (C) 2022 Adam Lecko et al. This is an open access article distributed under the Creative Commons Attribution License, which permits unrestricted use, distribution, and reproduction in any medium, provided the original work is properly cited.

In the present exploration, the authors define and inspect a new class of functions that are regular in the unit disc $\mathfrak{D}:=\{\varsigma \in \mathbb{C}$ $:|\varsigma|<1\}$, by using an adapted version of the interesting analytic formula offered by Robertson (unexploited) for starlike functions with respect to a boundary point by subordinating to an exponential function. Examples of some new subclasses are presented. Initial coefficient estimates are specified, and the familiar Fekete-Szegö inequality is obtained. Differential subordinations concerning these newly demarcated subclasses are also established.

\section{Introduction and Preliminary Results}

Let $\mathscr{H}$ be the class comprising of all holomorphic functions in the unit disc $\mathfrak{D}:=\{\varsigma \in \mathbb{C}:|\varsigma|<1\}$. Also, let $\mathscr{A}$ signify the subclass of $\mathscr{H}$ entailing of functions $h \in \mathscr{A}$ be of the form

$$
h(\varsigma)=\varsigma+\sum_{n=2}^{\infty} a_{n} \varsigma^{n}, \quad \varsigma \in \mathfrak{D},
$$

with the normalization $h(0)=h^{\prime}(0)-1=0$. Denote by $\mathcal{S}$, the subclass of $\mathscr{A}$ comprising univalent functions. Two conversant subclasses of $\mathscr{A}$ are familiarized by Robertson [1], are defined with their analytical description as

$$
\begin{aligned}
& \mathcal{S}^{*}(\alpha):=\left\{h \in \mathscr{A}: \mathfrak{R}\left(\frac{\varsigma h^{\prime}(\varsigma)}{h(\varsigma)}\right)>\alpha, \quad \varsigma \in \mathfrak{D}\right\}, \\
& \mathscr{C}(\alpha):=\left\{h \in \mathscr{A}: \mathfrak{R}\left(1+\frac{\varsigma h^{\prime \prime}(\varsigma)}{h^{\prime}(\varsigma)}\right)>\alpha, \quad \varsigma \in \mathfrak{D}\right\},
\end{aligned}
$$

and are correspondingly known as starlike and convex functions of order $\alpha(0 \leq \alpha<1)$. It is well known that $\mathcal{S}^{*}(\alpha) \subset \mathcal{S}$ and $\mathscr{C}(\alpha) \subset \mathcal{S}$. In interpretation of Alexander's relation, $h$ $\in \mathscr{C}(\alpha) \Leftrightarrow \varsigma h^{\prime}(\varsigma) \in \mathcal{S}^{*}(\alpha)$ for $\varsigma \in \mathfrak{D}$. For $\alpha=0$, the class $\mathcal{S}^{*}$ $:=\mathcal{S}^{*}(0)$ condenses to the well-known class of normalized starlike univalent functions, and $\mathscr{C}:=\mathscr{C}(0)$ reduces to the normalized convex univalent functions.

A function $f \in \mathscr{H}$ is subordinate to $\mathrm{g} \in \mathscr{H}$ written as $f$ $\prec g$ if there exists $\omega \in \mathscr{H}$ with $\omega(0)=0$ and $\omega(\mathfrak{D}) \subset \mathfrak{D}$ such that $f(\varsigma)=g(\omega(\varsigma))$ for every $\varsigma \in \mathfrak{D}$. In precise, if $g$ is univalent, then $f \prec g$ if and only if $f(0)=g(0)$ and $f(\mathfrak{D}) \subset g(\mathfrak{D})$.

Let $\mathscr{P}$ symbolize the class of functions $p \in \mathscr{H}$ with the normalization $p(0)=1$, i.e., of the form

$$
p(\varsigma)=1+\sum_{n=1}^{\infty} p_{n} \varsigma^{n}, \quad \varsigma \in \mathfrak{D}
$$

and such that $\mathfrak{R} p(\varsigma)>0$ for $\varsigma \in \mathfrak{D}$. Functions in $\mathscr{P}$ are called familiarly as the Carathéodory class of functions. Ma and Minda [2] proposed a appropriate subclass of $\mathscr{P}$ denoted 
by $\mathscr{P}^{*}(1)$ comprising of all $\Phi$ that is univalent in $\mathfrak{D}$ with

$$
\Phi(0)=1 ; \Phi^{\prime}(0)>0
$$

$\Phi(\mathfrak{D})$ is symmetric with respect to the real axis

(2) Starlike with respect to 1

He also represented the class $\Phi \in \mathscr{P}^{*}(1)$ by

$$
\Phi(\varsigma)=1+\sum_{n=1}^{\infty} B_{n} \varsigma^{n}, B_{1}>0 ; \varsigma \in \mathfrak{D}
$$

The class $\mathscr{P}^{*}(1)$ plays a vital part in defining generalized form of holomorphic functions. Ma and Minda [2] considered the function $\Phi \in \mathscr{P}^{*}(1)$ and defined $\mathcal{S}^{*}(\Phi)$ as the class of all $h \in \mathscr{A}$ such that $\varsigma h^{\prime}(\varsigma) / h(\varsigma) \prec \Phi(\varsigma)$ for $\varsigma \in \mathfrak{D}$. The above functions defined are called as functions of $\mathrm{Ma}$ and Minda kind. Observe that $\mathcal{S}^{*}(\alpha)=\mathcal{S}^{*}(\Phi)$ with $\Phi(\varsigma)=(1+$ $(1-2 \alpha) \varsigma) /(1-\varsigma), \varsigma \in \mathfrak{D}$.

There are recent articles ([3-6]) where subclasses of $\mathscr{A}$ were defined by using subordination satisfying the relation $\varsigma h^{\prime}(\varsigma) / h(\varsigma) \prec \Phi(\varsigma)$ for $\varsigma \in \mathfrak{D}$ (see also $[7,8]$ ). In particular, the exponential function $\Phi_{e}(\varsigma)=\mathrm{e}^{\varsigma}:=\exp (\varsigma)$, an entire function in $\mathbb{C}$ has positive real part in $\mathfrak{D}, \Phi_{e}(0)=1$, $\Phi_{e}^{\prime}(0)=1$, and $\Phi_{e}(\mathfrak{D})=\{w \in \mathbb{C}:|\log w|<1\}$, is symmetric with respect to the real axis and starlike with respect to 1 . Further, $\Phi_{e} \in \mathscr{P}^{*}(1)$ and therefore, it is now to make a remark that the class

$$
\mathcal{S}_{e}=\left\{f \in \mathscr{A}: \frac{\varsigma f^{\prime}(\varsigma)}{f(\varsigma)} \prec \Phi_{e}(\varsigma)=\mathrm{e}^{\varsigma}, \quad \varsigma \in \mathfrak{D}\right\}
$$

is well defined. For an attractive study on starlike functions connected with the exponential function, an individual can refer to Mendiratta et al. [9, 10] (see also the works of [11-13]).

We recall the class of close-to-convex functions denoted by $\mathscr{K}$ introduced and studied by Kaplan [14]. A function $h$ $\in \mathscr{H}$ is called to be close-to-convex if and only if there exist a function $\psi \in \mathscr{C}$ and $\beta \in(-\pi / 2, \pi / 2)$ such that

$$
\mathfrak{R}\left(\frac{\mathrm{e}^{\mathrm{i} \beta} h^{\prime}(\varsigma)}{\psi^{\prime}(\varsigma)}\right)>0, \quad \varsigma \in \mathfrak{D}
$$

Remarking at this time that even though starlikeness of a fixed order has been discussed and well thought-out in detail in countless articles in excess of a elongated stage of period, class of univalent functions $g \in \mathscr{H}$ that maps $\mathfrak{D}$ onto $\Omega$, starlike domain with reverence to a boundary point is still a conception that is not exclusively explored. Robertson [15] recognized this examination and introduced a new subclass

$$
\mathscr{G}^{*}=\left\{g \in \mathscr{H}: \mathfrak{R}\left(\mathrm{e}^{\mathrm{i} \delta} g(\varsigma)\right)>0 ; \delta \in \mathbb{R} ; \forall \varsigma \in \mathfrak{D}\right\},
$$

with

$$
g(0)=1, \quad g(1):=\lim _{r \longrightarrow 1^{-}} g(r)=0,
$$

and maps (univalently) $\mathfrak{D}$ onto a domain starlike with respect to the origin. Presume in addition that the constant function $g \equiv 1 \in \mathscr{G}^{*}$, in addition, Robertson through a conjecture that $\mathscr{G}^{*}$ coincides with the class $\mathscr{G}$ of all $g \in \mathscr{H}$ of the structure

$$
g(\varsigma)=1+\sum_{n=1}^{\infty} \vartheta_{n} \varsigma^{n}, \quad \varsigma \in \mathfrak{D}
$$

such that

$$
\mathfrak{R}\left(\frac{2 \varsigma \mathcal{g}^{\prime}(\varsigma)}{g(\varsigma)}+\frac{1+\varsigma}{1-\varsigma}\right)>0, \quad \varsigma \in \mathfrak{D}
$$

proving that $\mathscr{G} \subset \mathscr{G}^{*}$. Definitely, in the same article Robertson shown that if $g \in \mathscr{G}$ and $g \neq 1$, then $g \in \mathscr{K}$ and so univalent in $\mathfrak{D}$. It is importance of citing that (11) was identified by much erstwhile by Styer [16]. This surmise of Robertson that $\mathscr{G}^{*}$ coincide with the class $\mathscr{G}$ was soon after proved by Lyzzaik [17], where he established that $\mathscr{G}^{*} \subset \mathscr{G}$.

A different analytical categorization of starlike functions with respect to a boundary point was proposed by Lecko [18] proving the necessity. The sufficiency part of the categorization was afterwards proved by Lecko and Lyzzaik [19] (see [[20], Chapter VII] as well). Encouraged by the article of Robertson [15], Aharanov et al. [21] (see also [22]) investigated about the class of functions that are sprirallike with respect to a boundary point. Let

$$
\mathrm{P}(\varsigma ; M):=\frac{4 \varsigma}{\left(\sqrt{(1-\varsigma)^{2}+4 \varsigma / M}+1-\varsigma\right)^{2}}, \sqrt{1}:=1, \quad \varsigma \in \mathfrak{D}
$$

be the Pick function. By using the Pick function $\mathrm{P}(\varsigma ; M)$, the author in [23] considered another closely related class to $\mathscr{G}$, the family $\mathscr{G}(M), M>1$, comprising of all $g \in \mathscr{H}$ of the form (10) such that

$$
\mathfrak{R}\left(\frac{2 \varsigma g^{\prime}(\varsigma)}{g(\varsigma)}+\frac{\varsigma \mathrm{P}^{\prime}(\varsigma ; M)}{\mathrm{P}(\varsigma ; M)}\right)>0, \quad \varsigma \in \mathfrak{D}
$$

In [24], Todorov established a structural formula and coefficient estimates by associating $\mathscr{G}$ with a functional $f(\varsigma$ )$/ 1-\varsigma$ for $\varsigma \in \mathfrak{D}$. For $g \in \mathscr{H}$ in (10), Obradovič and Owa [25] and Silverman and Silvia [26] separately introduced the classes

$$
\mathscr{G}_{\alpha}=\left\{\mathfrak{R}\left(\frac{\varsigma g^{\prime}(\varsigma)}{g(\varsigma)}+(1-\alpha) \frac{1+\varsigma}{1-\varsigma}\right)>0, \quad \varsigma \in \mathfrak{D}\right\},
$$


where $\alpha \in[0,1)$. The authors in [26] confirmed a remarkable fact that for each $\alpha \in[0,1)$, the class $\mathscr{G}_{\alpha}$ is a subclass of $\mathscr{G}^{*}$. Clearly, $\mathscr{G}_{1 / 2}=\mathscr{G}$ and appealing coefficient inequalities of $\mathscr{G}$ were established in [27].

For $g \in \mathscr{H}$ assumed as in (10) and $-1<E \leq 1 ;-E<F \leq 1$ , Jakubowski and Włodarczyk [28] defined the class $\mathscr{G}(E, F)$ as

$$
\mathfrak{R}(J(\varsigma))>0, \quad \varsigma \in \mathfrak{D},
$$

where

$$
J(\varsigma)=\frac{2 \varsigma g^{\prime}(\varsigma)}{g(\varsigma)}+\frac{1+E \varsigma}{1-F \varsigma} .
$$

By desirable quality of the initiative proposed in [2], Mohd and Darus in [29] presented a new class $\mathcal{S}_{b}^{*}(\Phi)$, where $\Phi \in \mathscr{P}^{*}(1)$, of all $g \in \mathscr{H}$ of the form (10) such that

$$
\frac{2 \varsigma g^{\prime}(\varsigma)}{g(\varsigma)}+\frac{1+\varsigma}{1-\varsigma} \prec \Phi(\varsigma), \quad \varsigma \in \mathfrak{D}
$$

An additional appealing class on the above direction was in recent times analyzed by Lecko et al. [30].

The most important intend of the present article is to illustrate and do a organized inquiry of the function class defined as below.

Definition 1. For $g \in \mathscr{H}$ and as assumed in (10), we let a new class $\mathscr{G}_{e}$ as

$$
\mathscr{G}_{e}=\left\{g \in \mathscr{H}: \frac{2 \varsigma g^{\prime}(\varsigma)}{g(\varsigma)}+\frac{1+\varsigma}{1-\varsigma} \prec \mathrm{e}^{\varsigma}, \quad \varsigma \in \mathfrak{D}\right\} .
$$

Remark 2. Note that the condition (18) is well defined, for

$$
p(\varsigma):=\frac{2 \varsigma g^{\prime}(\varsigma)}{g(\varsigma)}+\frac{1+\varsigma}{1-\varsigma}, \quad \varsigma \in \mathfrak{D}
$$

is holomorphic in $\mathfrak{D}$.

Based on the description of the class $\mathscr{G}_{e}$ and on the analytical characterization of the class $\mathscr{G}^{*}$ of starlike functions with respect to a boundary point, we can prepare the next result.

\section{Representation Theorem and Coefficient Results}

Let us start the section with the following representation theorem which in fact offers a handy procedure to build functions in our new class $\mathscr{G}_{e}$.
Theorem 3. A function $g \in \mathscr{G}_{e}$ if and only if there exists $p$ $\in \mathscr{H}$ such that $p<\Phi_{e}$ and

$$
g(\varsigma)=(1-\varsigma) \exp \left(\frac{1}{2} \int_{0}^{\varsigma} \frac{p(\zeta)-1}{\zeta} d \zeta\right), \quad \varsigma \in \mathfrak{D} .
$$

Proof. Let us suppose that $g \in \mathscr{G}_{e}$, then, a function $p$ defined by (19) is holomorphic and satisfies $p \prec \Phi_{e}$. Also, (19) can be rewritten in the type

$$
\frac{2 g^{\prime}(\varsigma)}{g(\varsigma)}+\frac{2}{1-\varsigma}=\frac{p(\varsigma)-1}{\varsigma}, \quad \varsigma \in \mathfrak{D} \text {. }
$$

This upon integration give

$$
\log \frac{(g(\varsigma))^{2}}{(1-\varsigma)^{2}}=\int_{0}^{\varsigma} \frac{p(\zeta)-1}{\zeta} d \zeta, \quad \varsigma \in \mathfrak{D}, \quad \log 1:=0
$$

This in essence gives

$$
(g(\varsigma))^{2}=(1-\varsigma)^{2} \exp \left(\int_{0}^{\varsigma} \frac{p(\zeta)-1}{\zeta} d \zeta\right), \quad \varsigma \in \mathfrak{D},
$$

which imply (20).

Let us presume $p \prec \Phi_{e}$. By defining a function $g$ as in (20), and by observing that $p(0)=1$, it is noticeable that $g$ is holomorphic in $\mathfrak{D}$. A working out shows that $g$ satisfies (21); so, (19). Thus, $g \in \mathscr{G}_{e}$, which ends the confirmation of the theorem.

Let $\Psi_{e}$ be a holomorphic function which is the solution of the differential equation (see also [[10], p. 367])

$$
\frac{\varsigma_{e}^{\prime}(\varsigma)}{\Psi_{e}(\varsigma)}=\mathrm{e}^{\varsigma}, \quad \varsigma \in \mathfrak{D}, \quad \Psi_{e}(0)=0, \quad \Psi_{e}^{\prime}(0)=1,
$$

i.e.,

$$
\begin{aligned}
\Psi_{e}(\varsigma)= & \varsigma \exp \left(\int_{0}^{\varsigma} \frac{e^{\zeta}-1}{\zeta} d \zeta\right)=\varsigma+\varsigma^{2} \\
& +\frac{3}{4} \varsigma^{3}+\frac{17}{36} \varsigma^{4}+\cdots, \quad \varsigma \in \mathfrak{D} .
\end{aligned}
$$

Next, we present few examples for the class $\mathscr{G}_{e}$.

\section{Example 4.}

(1) For a specified $A \in \mathbb{R}$ and $\varsigma \in \mathfrak{D}$, let us name

$$
\begin{aligned}
& p_{A}(\varsigma):=1+A \varsigma, \\
& g_{A}(\varsigma):=(1-\varsigma) \exp \left(\frac{A \varsigma}{2}\right), \quad \varsigma \in \mathfrak{D} .
\end{aligned}
$$


Note down that $g_{A} \in \mathscr{H}$ with $g_{A}(0)=1$. Observe that

$$
\frac{2 \varsigma g_{A^{\prime}}(\varsigma)}{g_{A}(\varsigma)}+\frac{1+\varsigma}{1-\varsigma}=p_{A}(\varsigma), \quad \varsigma \in \mathfrak{D} .
$$

We finish that $g_{A} \in \mathscr{G}_{e}$ for $|A| \leq 1-1 /$ e.

(2) Given $-1<A \leq 1$ and $-A<B<1$, define

$$
w=p_{A, B}(\varsigma):=\frac{1+A \varsigma}{1-B \varsigma}, \quad \varsigma \in \mathfrak{D} .
$$

Then, we identify that $p_{A, B}(\mathfrak{D})$ is an open disk symmetrical with respect to the real axis centered at $(1+A B) /(1-$ $\left.B^{2}\right)$ of radius $(A+B) /\left(1-B^{2}\right)$. In particular, for $B=A$, this disk is given by

$$
\left|w-\frac{1+A^{2}}{1-A^{2}}\right|<\frac{2 A}{1-A^{2}}
$$

with diametric end points $x_{L}:=(1-|A|) /(1+|A|)$ and $x_{R}$ $:=(1+|A|) /(1-|A|)$. Since $x_{L} \geq 1 / \mathrm{e}$ and $x_{R} \leq \mathrm{e}$ iff $|A| \leq(\mathrm{e}$ $-1) /(\mathrm{e}+1)$, we perceive that then $p_{A, A}<\Phi_{e}$. As a result, a function $g \in \mathscr{H}$ with $g(0)=1$ defined by

$$
\frac{2 \varsigma g^{\prime}(\varsigma)}{g(\varsigma)}+\frac{1+\varsigma}{1-\varsigma}=p_{A, A}(\varsigma), \quad \varsigma \in \mathfrak{D},
$$

i.e., the function

$$
g(\varsigma)=\frac{1-\varsigma}{1-A \varsigma}, \quad \varsigma \in \mathfrak{D},
$$

belongs to the class $\mathscr{G}_{e}$ for $|A| \leq(\mathrm{e}-1) /(\mathrm{e}+1)$.

Theorem 5. Let $0<r<1$. If $g \in \mathscr{G}_{e}$, then

(i)

$$
\sqrt{\frac{-\Psi_{e}(-r)}{r}}(1-r) \leq|g(\varsigma)| \leq \sqrt{\frac{\Psi_{e}(-r)}{r}}(1+r), \quad|\varsigma|=r .
$$

(ii)

$$
\left|\arg \frac{g\left(\varsigma_{0}\right)}{\left(1-\varsigma_{0}\right)^{2}}\right| \leq \frac{1}{2} \max _{|\varsigma|=r} \arg \frac{\Psi_{e}(\varsigma)}{\varsigma},\left|\varsigma_{0}\right|=r, \quad \arg 1:=0 .
$$

Proof. Let $g \in \mathscr{G}_{e}$.

(i) Describe the function

$$
h(\varsigma):=\frac{\varsigma(g(\varsigma))^{2}}{(1-\varsigma)^{2}}, \quad \varsigma \in \mathfrak{D}
$$

Obviously, $h$ is a holomorphic function in $\mathfrak{D}$, and an uncomplicated working out yields

$$
\frac{\varsigma h^{\prime}(\varsigma)}{h(\varsigma)}=\frac{2 \varsigma g^{\prime}(\varsigma)}{g(\varsigma)}+\frac{1+\varsigma}{1-\varsigma}, \quad \varsigma \in \mathfrak{D} .
$$

It is straightforward to witness from the above that $g \in$ $\mathscr{G}_{e}$ if and only if

$$
\frac{\varsigma h^{\prime}(\varsigma)}{h(\varsigma)} \prec e^{\varsigma}, \quad \varsigma \in \mathfrak{D} .
$$

By the result of Corollary $1^{\prime}$ of [2], we obtain

$$
-\Psi_{e}(-r) \leq|h(\varsigma)| \leq \Psi_{e}(r), \quad|\varsigma|=r,
$$

i.e., by using (34),

$$
-\Psi_{e}(-r) \leq\left|\frac{\varsigma(g(\varsigma))^{2}}{(1-\varsigma)^{2}}\right| \leq \Psi_{e}(r), \quad|\varsigma|=r,
$$

which gives (32).

(ii) By (36), a function $h$ defined by (34) belongs to $\delta^{*}$ $\left(\Phi_{e}\right)$. Due to Corollary $3^{\prime}$ of [2], the inequality

$$
\left|\arg \frac{h\left(\varsigma_{0}\right)}{\varsigma_{0}}\right| \leq \max _{|\varsigma|=r} \arg \frac{\Psi_{e}(\varsigma)}{\varsigma}, \quad\left|\varsigma_{0}\right|=r
$$

is valid. Using now (34) in turn yields (33)

Next, we ascertain some coefficient results for the class $g \in \mathscr{G}_{e}$. Let $\mathscr{B}:=\{\omega \in \mathscr{H}:|\omega(\varsigma)| \leq 1, \varsigma \in \mathfrak{D}\}$ and $\mathscr{B}_{0}$ be the subclass of $\mathscr{B}$ consisting of functions $\omega$ such that $\omega(0)=0$. We comment at this time that the elements of $\mathscr{B}_{0}$ are termed as Schwarz functions.

We will pertain two lemmas below to prove our main results.

Lemma 6. (see [2]). If $p \in \mathscr{P}$ is of the form (3), then for $\mu \in \mathbb{C}$,

$$
\left|p_{2}-\mu p_{1}^{2}\right| \leq 2 \max \{1,|2 \mu-1|\} .
$$

In particular, if $\mu$ is a real number, then

$$
\left|p_{2}-\mu p_{1}^{2}\right| \leq \begin{cases}-4 \mu+2, & \mu \leq 0, \\ 2, & 0 \leq \mu \leq 1, \\ 4 \mu-2, & \mu \geq 1 .\end{cases}
$$

When $\mu<0$ or $\mu>1$, the equality holds true if and only if $p(\varsigma)=(1+\varsigma) /(1-\varsigma)=: \mathscr{L}(\varsigma), \varsigma \in \mathfrak{D}$, or one of its rotations. If $0<\mu<1$, then the equality holds true if and only if $p(\varsigma$ $=\mathscr{L}\left(\varsigma^{2}\right), \varsigma \in \mathfrak{D}$, or one of its rotations. If $\mu=0$, the equality 
holds true if and only if

$$
p(\varsigma)=\frac{1}{2}(1+\lambda) \mathscr{L}(\varsigma)+\frac{1}{2}(1-\lambda) \mathscr{L}(-\varsigma), \quad \varsigma \in \mathfrak{D}
$$

where $0 \leq \lambda \leq 1$, or one of its rotations. If $\mu=1$, then the equality holds true if $p$ is a reciprocal of one of the functions such that the equality holds true in the case when $\mu=0$.

Lemma 7. (see [31]). If $p \in \mathscr{P}$ is of the form (3) and $\beta(2 \beta$ $-1) \leq \delta \leq \beta$, then

$$
\left|p_{3}-2 \beta p_{1} p_{2}+\delta p_{1}^{3}\right| \leq 2
$$

At the moment, we are in a position to state the theorem which give a few better bounds for early coefficients and the Fekete-Szegö inequalities for $f \in \mathscr{G}_{e}$.

Theorem 8. If $g \in \mathscr{G}_{e}$ is of the form (10), then

$$
\begin{gathered}
\left|\vartheta_{1}+1\right| \leq \frac{1}{2}, \\
\left|\vartheta_{1}\right| \leq \frac{3}{2}, \\
\left|2 \vartheta_{2}-\vartheta_{1}^{2}+1\right| \leq \frac{1}{2}, \\
\left|\vartheta_{2}\right| \leq \frac{3}{4}, \\
\left|3 \vartheta_{3}-3 \vartheta_{1} \vartheta_{2}+\vartheta_{1}^{3}+1\right| \leq \frac{1}{2},
\end{gathered}
$$

and for $\delta \in \mathbb{R}$,

$$
\left|\vartheta_{2}-\delta \vartheta_{1}^{2}\right| \leq \frac{1}{4}(\max \{1,|\delta-1|\}+2|2 \delta-1|+4|\delta|) .
$$

Inequalities (44), (45), (46), (47), and (48) are sharp.

Proof. In view of (18), there exists $\omega \in \mathscr{B}_{0}$ such that

$$
\frac{2 \varsigma g^{\prime}(\varsigma)}{g(\varsigma)}+\frac{1+\varsigma}{1-\varsigma}=\Phi_{e}(\omega(\varsigma))=\exp (\omega(\varsigma)), \quad \varsigma \in \mathfrak{D}
$$

By an application of (10), one can easily obtain with simple computation that

$$
\begin{aligned}
\frac{2 \varsigma g^{\prime}(\varsigma)}{g(\varsigma)}+\frac{1+\varsigma}{1-\varsigma}= & +2\left(\vartheta_{1}+1\right) \varsigma+2\left(2 \vartheta_{2}-\vartheta_{1}^{2}+1\right) \varsigma^{2} \\
& +2\left(3 \vartheta_{3}-3 \vartheta_{1} \vartheta_{2}+\vartheta_{1}^{3}+1\right) \varsigma^{3}+\cdots, \quad \varsigma \in \mathfrak{D} .
\end{aligned}
$$

Define the function $p$ by

$$
p(\varsigma)=\frac{1+\omega(\varsigma)}{1-\omega(\varsigma)}=1+p_{1} \varsigma+p_{2} \varsigma^{2}+\cdots, \quad \varsigma \in \mathfrak{D} .
$$

Clearly, $p \in \mathscr{P}$. Moreover,

$$
\begin{aligned}
\omega(\varsigma)= & \frac{p(\varsigma)-1}{p(\varsigma)+1}=\frac{p_{1}}{2} \varsigma+\left(\frac{p_{2}}{2}-\frac{p_{1}^{2}}{4}\right) \varsigma^{2} \\
& +\left(\frac{p_{3}}{2}-\frac{p_{1} p_{2}}{2}+\frac{p_{1}^{3}}{8}\right) \varsigma^{3}+\cdots, \quad \varsigma \in \mathfrak{D} .
\end{aligned}
$$

Hence,

$$
\begin{aligned}
\exp (\omega(\varsigma))=1 & +\omega(\varsigma)+\frac{(\omega(\varsigma))^{2}}{2}+\frac{(\omega(\varsigma))^{3}}{6}+\cdots=1+\frac{p_{1} \varsigma}{2} \\
& +\left(\frac{p_{2}}{2}-\frac{p_{1}^{2}}{8}\right) \varsigma^{2}+\left(\frac{p_{3}}{2}-\frac{p_{1} p_{2}}{4}+\frac{p_{1}^{3}}{48}\right) \varsigma^{3}+\cdots, \quad \varsigma \in \mathfrak{D} .
\end{aligned}
$$

Substituting (51) and (54) into (50), by comparing the corresponding coefficients, we obtain

$$
\begin{gathered}
2\left(\vartheta_{1}+1\right)=\frac{p_{1}}{2}, \\
2\left(2 \vartheta_{2}-\vartheta_{1}^{2}+1\right)=\frac{p_{2}}{2}-\frac{p_{1}^{2}}{8}, \\
2\left(3 \vartheta_{3}-3 \vartheta_{1} \vartheta_{2}+\vartheta_{1}^{3}+1\right)=\frac{p_{3}}{2}-\frac{p_{1} p_{2}}{4}+\frac{p_{1}^{3}}{48} .
\end{gathered}
$$

Since (e.g., ([[32]], Vol. I, p. 80)),

$$
\left|p_{n}\right| \leq 2, \quad n \in \mathbb{N} \text {. }
$$

From (55), we obtain (44). Rewriting (55) as $\vartheta_{1}=p_{1} / 4-1$, (45) easily follows. Further, (56) together with (40) yields

$$
\left|2\left(2 \vartheta_{2}-\vartheta_{1}^{2}+1\right)\right|=\left|\frac{p_{2}}{2}-\frac{p_{1}^{2}}{8}\right| \leq 1,
$$

which proves (46).

Upon applying (55) for $\vartheta_{1}$ in (56), we get

$$
4 \vartheta_{2}=\frac{p_{2}}{2}-p_{1}
$$

Hence, by applying (41), we obtain (47). An application of (43) in (57) gives

$$
\left|6 \vartheta_{3}-6 \vartheta_{1} \vartheta_{2}+2 \vartheta_{1}^{3}+2\right|=\left|\frac{p_{3}}{2}-\frac{p_{1} p_{2}}{4}+\frac{p_{1}^{3}}{48}\right| \leq 1,
$$

i.e., the inequality (48). 
Using (60) and making use of the expression for $\vartheta_{1}$ and in turn by applying (41) and (58), we get

$$
\left|\vartheta_{2}-\delta \vartheta_{1}^{2}\right| \leq \frac{1}{8}\left(\left|p_{2}-\frac{\delta}{2} p_{1}^{2}\right|+2|2 \delta-1|\left|p_{1}\right|+8|\delta|\right), \quad \delta \in \mathbb{R}
$$

which leads to the inequality (49).

Equalities in (44) and (45) hold for the function $p=\mathscr{L}$; in (46) for the function $p(\varsigma)=\mathscr{L}\left(\varsigma^{2}\right), \varsigma \in \mathfrak{D}$, in (47) for the function $p(\varsigma)=\mathscr{L}(-\varsigma), \varsigma \in \mathfrak{D}$ and in (48) for the function $p$ $(\varsigma)=\mathscr{L}\left(\varsigma^{3}\right), \varsigma \in \mathfrak{D}$.

\section{Differential Subordination Results Involving $\mathscr{G}_{e}$}

In this segment, we derive certain differential subordination result concerning the class $\mathscr{G}_{e}$.

To demonstrate differential subordination results, we recollect the next lemma (see ([[33]], Theorem $8.4 \mathrm{~h}$, p. 132)).

$Q$ is starlike univalent in $\mathfrak{D}$, or

$h$ is convex univalent in $\mathfrak{D}$

Lemma 9. Suppose $q$ is univalent in $\mathfrak{D}, \theta$ and $\varphi$ be holomorphic in a domain D containing $q(\mathfrak{D})$ with $\varphi(w) \neq 0$ when $w \in q(\mathfrak{D})$. Let $Q(\varsigma):=\varsigma q^{\prime}(\varsigma) \varphi(q(\varsigma))$ and $h(\varsigma):=\theta(q(\varsigma))+Q$ ( $)$ for $\varsigma \in \mathfrak{D}$. Suppose that either

Assume also that

(iii)

$$
\mathfrak{R} \frac{\varsigma h^{\prime}(\varsigma)}{Q(\varsigma)}>0, \quad \varsigma \in \mathfrak{D}
$$

If $p \in \mathscr{H}$ with $p(0)=q(0), p(\mathfrak{D}) \subset D$, and

$\theta(p(\varsigma))+\varsigma p^{\prime}(\varsigma) \varphi(p(\varsigma)) \prec \theta(q(\varsigma))+\varsigma q^{\prime}(\varsigma) \varphi(q(\varsigma)), \quad \varsigma \in \mathfrak{D}$,

then $p \prec q$ and $q$ are the best dominant.

Theorem 10. Let $g \in \mathscr{H}$ and $g(0)=1$. If $g$ satisfies the subordination condition,

$$
\frac{2 \varsigma g^{\prime}(\varsigma)}{g(\varsigma)}+\frac{1+\varsigma}{1-\varsigma} \prec 1+\varsigma, \quad \varsigma \in \mathfrak{D}
$$

Then,

$$
p(\varsigma):=\frac{(g(\varsigma))^{2}}{(1-\varsigma)^{2}} \prec e^{\varsigma}, \quad \varsigma \in \mathfrak{D}
$$

Proof. Let $D:=\mathbb{C} \backslash\{0\}$. Let $\theta(w):=1, w \in \mathbb{C}$ and $\varphi(w):=1 /$ $w, w \in D$. Note that $\Phi_{e}(\mathfrak{D}) \subset D$ and $\theta$ and $\varphi$ are holomorphic in $D$. Thus,

$$
Q(\varsigma):=\varsigma \Phi_{e}^{\prime}(\varsigma) \varphi\left(\Phi_{e}(\varsigma)\right)=\frac{\varsigma \Phi_{e}{ }^{\prime}(\varsigma)}{\Phi_{e}(\varsigma)}=\varsigma, \quad \varsigma \in \mathfrak{D}
$$

is well defined and holomorphic. Clearly, $Q$ is a univalent starlike function and so for a function $h(\varsigma):=\theta\left(\Phi_{e}(\varsigma)\right)+Q$ $(\varsigma)=1+Q(\varsigma), \varsigma \in \mathfrak{D}$, we achieve

$$
\mathfrak{R} \frac{\varsigma h^{\prime}(\varsigma)}{Q(\varsigma)}=\mathfrak{R} \frac{\varsigma Q^{\prime}(\varsigma)}{Q(\varsigma)}=1>0, \quad \varsigma \in \mathfrak{D} .
$$

Hence, for any function $p$ belonging to $\mathscr{H}$ with $p(0)=$ $\Phi_{e}(0)=1$ such that $p(\mathfrak{D}) \subset D$, i.e., for $p$ nonvanishing in $\mathfrak{D}$ , by applying Lemma 9 , we infer that from the subordination

$$
1+\frac{\varsigma p^{\prime}(\varsigma)}{p(\varsigma)} \prec 1+\frac{\varsigma \Phi_{e^{\prime}}(\varsigma)}{\Phi_{e}(\varsigma)}=1+\varsigma, \quad \varsigma \in \mathfrak{D},
$$

it follows the subordination $p \prec \Phi_{e}$.

Next, we at this time take $g \in \mathscr{H}$ with $g(0)=1$ and $g(\varsigma)$ be nonzero for $\varsigma \in \mathfrak{D}$ satisfying (65). Let a function $p$ be taken as in (66). Then, one can notice that $p(0)=\Phi_{e}(0)=1$ , $p(\varsigma) \neq 0$, for $\varsigma \in \mathfrak{D}$, and $p$ is holomorphic. Since

$$
1+\frac{\varsigma p^{\prime}(\varsigma)}{p(\varsigma)}=\frac{2 \varsigma g^{\prime}(\varsigma)}{g(\varsigma)}+\frac{1+\varsigma}{1-\varsigma}, \quad \varsigma \in \mathfrak{D},
$$

from (69), the conclusion (66) follows, which complete the proof.

Theorem 11. Let $g \in \mathscr{H}$ with $g(0)=1$. If $g$ satisfies

$$
\frac{2 \varsigma g^{\prime}(\varsigma)}{g(\varsigma)}+\frac{1+\varsigma}{1-\varsigma} \prec e^{\varsigma}+\varsigma, \quad \varsigma \in \mathfrak{D},
$$

then

$$
p(\varsigma):=\varsigma\left(\frac{g(\varsigma)}{1-\varsigma}\right)^{2}\left(\int_{0}^{\varsigma}\left(\frac{g(\zeta)}{1-\zeta}\right)^{2} d \zeta\right)^{-1} \prec e^{\varsigma}, \quad \varsigma \in \mathfrak{D} \text {. }
$$

Proof. Let $D:=\mathbb{C} \backslash\{0\}$. Let $\phi(w):=w, w \in \mathbb{C}$, and $\psi(w):=1$ $/ w, w \in D$. Note that $\Phi_{e}(\mathfrak{D}) \subset D$ and $\phi$ and $\psi$ are holomorphic in $D$. Thus, the function $Q$ defined by (67), i.e., the identity function, is univalent starlike. Hence, for a function $h(\varsigma):=\theta\left(\Phi_{e}(\varsigma)\right)+Q(\varsigma)=\Phi_{e}(\varsigma)+Q(\varsigma), \varsigma \in \mathfrak{D}$, we obtain

$$
\begin{aligned}
\mathfrak{R} \frac{\varsigma h^{\prime}(\varsigma)}{Q(\varsigma)} & =\mathfrak{R} \frac{\varsigma \Phi_{e^{\prime}}(\varsigma)}{Q(\varsigma)}+\mathfrak{R} \frac{\varsigma Q^{\prime}(\varsigma)}{Q(\varsigma)} \\
& =\mathfrak{R} \Phi_{e}(\varsigma)+\mathfrak{R} \frac{\varsigma Q^{\prime}(\varsigma)}{Q(\varsigma)}>0, \quad \varsigma \in \mathfrak{D} .
\end{aligned}
$$

Thus, for any function $p \in \mathscr{H}$ with $p(0)=\Phi_{e}(0)=1$ such 
that $p(\mathfrak{D}) \subset D$, i.e., $p(\varsigma) \neq 0$ for $\varsigma \in \mathfrak{D}$, by applying Lemma 9 , we deduce that from the subordination

$$
p(\varsigma)+\frac{\varsigma p^{\prime}(\varsigma)}{p(\varsigma)} \prec \Phi_{e}(\varsigma)+\frac{\varsigma \Phi_{e^{\prime}}(\varsigma)}{\Phi_{e}(\varsigma)}=\mathrm{e}^{\varsigma}+\varsigma, \quad \varsigma \in \mathfrak{D},
$$

it follows the subordination $p<\Phi_{e}$.

Let now take $g \in \mathscr{H}$ with $g(0)=1$ and $g(\varsigma) \neq 0$ for $\varsigma \in \mathfrak{D}$ satisfying (65). Define a function $p$ as in (72). We see that

$$
\begin{aligned}
p(0) & =\lim _{\varsigma \longrightarrow 0} \varsigma\left(\frac{g(\varsigma)}{1-\varsigma}\right)^{2}\left(\int_{0}^{\varsigma}\left(\frac{g(\zeta)}{1-\zeta}\right)^{2} d \zeta\right)^{-1} \\
& =(g(0))^{2} \lim _{\varsigma \longrightarrow 0} \varsigma\left(\int_{0}^{\varsigma}\left(\frac{g(\zeta)}{1-\zeta}\right)^{2} d \zeta\right)^{-1}=1=\Phi_{e}(0)
\end{aligned}
$$

$p(\varsigma)=0$ for $\varsigma \in \mathfrak{D}$ and $p$ is holomorphic. Since

$$
p(\varsigma)+\frac{\varsigma p^{\prime}(\varsigma)}{p(\varsigma)}=\frac{2 \varsigma g^{\prime}(\varsigma)}{g(\varsigma)}+\frac{1+\varsigma}{1-\varsigma}, \quad \varsigma \in \mathfrak{D},
$$

from (74), (71) follows which completes the proof.

\section{Data Availability}

No data sets were used.

\section{Conflicts of Interest}

The authors declare that they have no conflicts of interest.

\section{Acknowledgments}

The work of Dr. S. Sivasubramanian is supported by a grant from the Science and Engineering Research Board, Government of India under Mathematical Research Impact Centric Support of Department of Science and Technology (DST) vide ref: MTR/2017/000607.

\section{References}

[1] M. S. Robertson, "On the theory of univalent functions," The Annals of Mathematics, vol. 37, no. 2, pp. 374-408, 1936.

[2] W. C. Ma and D. Minda, "A unified treatment of some special classes of unvalent functions," Proceedings of the Conference on Complex Analysis (Tianjin; June 19-23, 1992), Z. Li, F. Ren, L. Yang, and S. Zhang, Eds., , pp. 157-169, International Press, Cambridge, Massachusetts, 1994.

[3] J. Dziok, R. K. Raina, and J. Sokól, “On $\alpha$-convex functions related to shell-like functions connected with Fibonacci numbers," Applied Mathematics and Computation, vol. 218, no. 3, pp. 996-1002, 2011.

[4] J. Dziok, R. K. Raina, and J. Sokół, “Certain results for a class of convex functions related to a shell-like curve connected with Fibonacci numbers," Computers \& Mathematcs with Applications, vol. 61, no. 9, pp. 2605-2613, 2011.

[5] J. Dziok, R. K. Raina, and J. Sokół, “On a class of starlike functions related to a shell-like curve connected with Fibonacci numbers," Mathematical and Computer Modelling, vol. 57, no. 5-6, pp. 1203-1211, 2013.

[6] J. Dziok, R. K. Raina, and J. Sokół, “Differential subordinations and $\alpha$-convex functions," Scientia, vol. 33, no. 3, pp. 609-620, 2013.

[7] R. K. Raina and J. Sokól, "Some properties related to a certain class of starlike functions," Comptes Rendus Mathematique, vol. 353, no. 11, pp. 973-978, 2015.

[8] R. K. Raina and J. Sokól, "Quelques proprietes liees a une classe de fonctions etoilees," Comptes Rendus Mathematique, vol. 353, no. 11, pp. 973-978, 2015.

[9] K. Khatter, V. Ravichandran, and S. S. Kumar, "Starlike functions associated with exponential function and the lemniscate of Bernoulli," Revista de la Real Academia de Ciencias Exactas, Físicas y Naturales. Serie A. Matemáticas, vol. 113, no. 1, pp. 233-253, 2019.

[10] R. Mendiratta, S. Nagpal, and V. Ravichandran, "On a subclass of strongly starlike functions associated with exponential function," Bulletin of the Malaysian Mathematical Sciences Society, vol. 38, no. 1, pp. 365-386, 2015.

[11] S. Çakmak, E. Yaşar, and S. Yalçın, "Convolutions of harmonic mappings convex in the horizontal direction," Journal of Function Spaces, vol. 2021, Article ID 2949573, 9 pages, 2021.

[12] G. Murugusundaramoorthy, A. Shakeel, and M. A. Kutbi, "Coefficient bounds of Kamali-type starlike functions related with a Limacon- shaped domain," Journal of Function Spaces, vol. 2021, Article ID 4395574, 10 pages, 2021.

[13] A. E. Shammaky and T. M. Seoudy, "Certain subclass of -valent functions associated with a new extended Ruscheweyh operator related to conic domains," Journal of Function Spaces, vol. 2021, Article ID 7298104, 8 pages, 2021.

[14] W. Kaplan, "Close-to-convex schlicht functions," The Michigan Mathematical Journal, vol. 1, no. 2, pp. 169-185, 1952.

[15] M. S. Robertson, "Univalent functions starlike with respect to a boundary point," Journal of Mathematical Analysis and Applications, vol. 81, no. 2, pp. 327-345, 1981.

[16] D. Styer, “On weakly starlike multivalent functions," Journal d'Analyse Mathématique, vol. 26, no. 1, pp. 217-233, 1973.

[17] A. Lyzzaik, "On a conjecture of M. S. Robertson," Proceedings of the American Mathematical Society, vol. 91, no. 1, pp. 108$110,1984$.

[18] A. Lecko, "On the class of functions starlike with respect to a boundary point," Journal of Mathematical Analysis and Applications, vol. 261, no. 2, pp. 649-664, 2001.

[19] A. Lecko and A. Lyzzaik, "A note on univalent functions starlike with respect to a boundary point," Journal of Mathematical Analysis and Applications, vol. 282, no. 2, pp. 846-851, 2003.

[20] A. Lecko, Some Methods in the Theory of Univalent Functions, Oficyna Wdawnicza Politechniki Rzeszowskiej, Rzeszów, 2005.

[21] D. Aharonov, M. Elin, and D. Shoikhet, "Spiral-like functions with respect to a boundary point," Journal of Mathematical Analysis and Applications, vol. 280, no. 1, pp. 17-29, 2003.

[22] A. Lecko, " $\delta$-Spirallike functions with respect to a boundary point," The Rocky Mountain Journal of Mathematics, vol. 38, no. 3, pp. 979-992, 2008.

[23] Z. J. Jakubowski, "On properties of the pick function and some applications of them," Acta Universitatis Purkynianae, vol. 42, pp. 51-62, 1999. 
[24] P. G. Todorov, "On the univalent functions starlike with respect to a boundary point," Proceedings of the American Mathematical Society, vol. 97, no. 4, pp. 602-604, 1986.

[25] M. Obradović and S. Owa, "On some classes of close-toconvex functions and its applications," Bulletin of the Institute of Mathematics, Academia Sinica, vol. 16, no. 2, pp. 123-133, 2012.

[26] H. Silverman and E. M. Silvia, "Subclasses of univalent functions starlike with respect to a boundary point," Houston Journal of Mathematics, vol. 16, no. 2, pp. 289-299, 1990.

[27] A. S. Abdullah, R. M. Ali, and V. Singh, "On functions starlike with respect to a boundary point," Annales Universitatis Mariae Curie - Sklod0wska Lublin - Polonia, vol. 50, pp. 7-15, 1996.

[28] Z. J. Jakubowski and A. Włodarczyk, "On some classes of functions of Robertson type," Annales Universitatis Mariae Curie Sklod0wska Lublin - Polonia, vol. LIX, pp. 27-42, 2005.

[29] M. H. Mohd and M. Darus, "Starlike function with respect to a boundary point defined by subordination," Advances in Mathematics: Scientific Journal, vol. 1, no. 1, pp. 15-21, 2012.

[30] A. Lecko, G. Murugusundaramoorthy, and S. Sivasubramanian, "On a class of analytic functions related to Robertson's formula and subordination," Boletín de la Sociedad Matemática Mexicana, vol. 27, no. 1, 2021.

[31] R. M. Ali, "Coefficients of the inverse of strongly starlike functions," Bulletin of the Malaysian Mathematical Sciences Society, vol. 26, 2003.

[32] A. W. Goodman, Univalent Functions, Mariner, Tampa, Florida, 1983.

[33] S. S. Miller and P. T. Mocanu, Differential Subordinations, Theory and Applications, Dekker, Basel, 2000. 